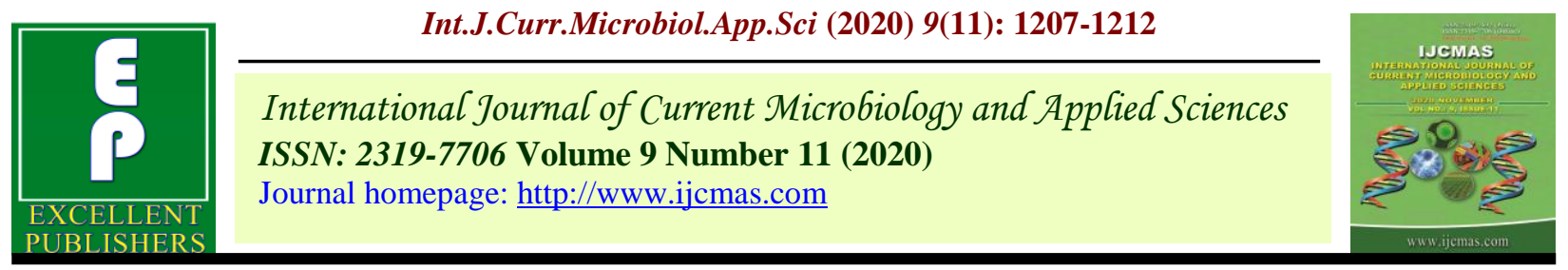

Original Research Article

https://doi.org/10.20546/ijcmas.2020.911.141

\title{
Physico-chemical Variability in Clone of Mango cv Langra at Kymore Plateau and Satpura Hills of Madhya Pradesh
}

\author{
Megha Rajpoot", T. R. Sharma, S. K. Pandey and Mohni Parmar \\ Department of Horticulture, Jawaharlal Nehru Krishi Vishwa Vidyalaya, \\ Jabalpur (M.P.), India \\ *Corresponding author
}

\begin{abstract}
A B S T R A C T
Keywords

Morphological,

Biochemical,

Clone, Langra

Article Info

Accepted:

10 October 2020

Available Online:

10 November 2020

Forty clones of Langra variety of mango were selected for study the morphological and biochemical variability at Fruit Research Station, Imalia, Jawaharlal Nehru KrishiVishwaVidyalaya, Jabalpur (M.P.). Significant variation among the clone was observed for all phyico-chemical traits under study, the maximum fruit length ( 9.45 $\mathrm{cm})$, width $(6.31 \mathrm{~cm})$ and fruit weight $(256.36 \mathrm{~g})$ was observed in cloneL-57 among all the clones. But yield was found in L-60 $(72.9 \mathrm{~kg})$, and maximum pulp weight found in L-43 (109.53 g). Maximum TSS was found in L-20 $\left(23.96{ }^{0}\right.$ Brix $)$, followed by L-70 (23.93 ${ }^{0}$ Brix). These results shows the significant level of genetic variation exists among 40 clones of mango cv.'Langra' which can be used for mass multiplication of superior clone and can be further utilized in breeding programs.
\end{abstract}

\section{Introduction}

Mango is one of the most important fruit crops of India after banana and referred to as "King of Fruits" (Singh, 1996), because of its delicious taste, excellent flavour and attractive fragrance. Mango is delicious fruit, but also has a great degree of nutritive value. Mango contains essential vitamins and dietary minerals. Mango can be grown under both tropical and sub-tropical climates. Mango is having winder adaptability with respect to soil, climate and altitude for its successful cultivation. It requires good rainfall during its growing season (June-October) and rainless dry weather from number on wards.
Temperature, rainfall, wind velocity and altitude are the main climatic factors which influence its growth and fruiting.

India ranks first among world mango producing countries. It is commonly found in India and cultivated an area of 2.258 million ha with an annual production of 21.822 million metric tons and productivity 9.66 MT/ha (Anon., 2018). Today world needs to increase crop productivity for the development of valuable varieties to changing environmental and biological challenges that meets to evolve the needs of local communities. Mango has rich varietal diversity and there are about 1600 varieties in 
the world. In India, there are hundreds of mango cultivars, of which about 50 varieties of mango are of commercial importance. Langra' is one of the leading commercial mango varieties of Northern India including Madhaya Pradesh which is known for mildly fibrous flesh and with a distinct pleasant taste and turpentine flavour. Significant variation exists among the clones of 'Langra' mango with respect to fruit size, shape, colour, quality and taste. Several studies have been made on characterization of intra-varietal variability of many different cultivars of mango. However, clonal variability studies in 'Langra' mango are limited. In the light of aim present study will be initiated to study the extent of clonal variability exists in 'Langra' mango variety using morphological and biochemical traits.

\section{Materials and Methods}

The experiment was carried out during 20182019 and 2019-2020 at Fruit Research Farm, Imalia, Jawaharlal Nehru Krishi Vishwa Vidyalaya, Jabalpur (M.P.) which is situated at $22^{\circ} 49^{\prime}$ and $20^{\circ} 80^{\prime}$ North latitude and $78^{\circ} 21^{\prime}$ and $80^{\circ} 58^{\prime}$ East longitude at an attitude of 411.78 meter above the mean sea level comes under Kymore plateau and Satpura hills" Agro-climatic Region of Madhya Pradesh. The climate of the region location was characterized by the long hot summer and cool winter. Dry and warm weather usually persists during the month of March to June and temperature may go as high as $44^{\circ} \mathrm{C}$ during these months. Rainfall is mainly concentrated in four months, starting from mid June to mid October. The average rainfall ranges from $1350 \mathrm{~mm}$, winter rains are also received usually. There is a probability of low rainfall after each four and five years. The soil of experimental site was clayey in texture (58.4\% clay, $22.5 \%$ silt and $20.1 \%$ sand) having $\mathrm{pH} 7.2$, medium available $\mathrm{N}$ (302 kgha-1), high in $\mathrm{P}$ (22.6 kg ha-1) and $\mathrm{K}$
(430.7 kg ha-1) with medium organic carbon $(0.70 \%)$. Forty healthy and vigorous superior clones from 50 year old plantation of 'Langra' were selected for the collection of data. Nine fruits were picked from each clone at harvest and then grouped into 3 lots (replicate) containing 3 fruits in each and analyzed in Randomized Block Design (RBD). Collected fruit samples were characterized and evaluated for variation in different quantitative traits viz. fruit length $(\mathrm{cm})$, fruit width $(\mathrm{cm})$, fruit weight $(\mathrm{g})$, pulp $(\%)$, peel $(\%)$, stone $(\%)$, yield $\left(\mathrm{Kg}\right.$ plant $\left.^{-1}\right)$, total soluble solids (TSS), ascorbic acids (mg/100g), acidity (\%), total sugar (\%), reducing sugar (\%), non-reducing sugar $(\%)$,were recorded. Peel and stone of each clone were carefully removed and cleaned with distilled water. TSS was recorded with refractometer. Acidity was determined by using standard titration method Ascorbic acid content and titratable acidity was determined by visual titration method (Ranganna, 2000). Ascorbic acid content of the juice was determined by titrating freshly extracted juice against 2, 6 Dichlorophenol indophenols dye Association of official Analytical Chemists. The reducing sugar was estimated by Lane and Eyon (1923). Copper titration methods as suggested by Association of Official Analytical Chemists (1975). Total sugars and reducing sugar was estimated as described by Lane and Eyon (1923). Quantitative measurement of TSS, acidity, ascorbic acid, total sugar, reducing sugar, non-reducing sugar was analyzed when fruit were naturally ripened.

\section{Results and Discussion}

The analysis of variance of 40 clones of 'Langra' identified in this investigation revealed significant differences (Table 1). In this study, the maximum fruit length $(9.46$ $\mathrm{cm}$ ) was observed in L-22 followed by L-57 $(9.45 \mathrm{~cm})$ and the minimum fruit length was 
observed in L-70 $(7.37 \mathrm{~cm})$. The highest fruit width was observed in L-62 $(6.45 \mathrm{~cm})$, followed by L-57, L-46, L-42, L-60, L-63, L$13, \mathrm{~L}-22$ and the lowest fruit width $(5.16 \mathrm{~cm})$ was found in L-35. L-57 recorded the highest fruit weight $(256.357 \mathrm{~g})$ but the minimum fruit weight was found in many mango clones. The pulp\% of L-43was found significantly superior compare to all other clones, here a huge difference between maximum (109.53) and minimum (38.63) value was found. The variation in this physical characteristic of mango fruit is natural due to differences in environmental and seasonal condition has also been observed earlier (Singh and Maurya, 1986; Badyal and Bhutani, 1989). The highest peel \% was found in L-20 (17.57) clone of Langra and lowest in L-21 (6.23).L-72 possess lowest stone percentage $(7.363 \%)$ whereas, L-68 possess maximum stone percentage $(20.52 \%)$. The highest fruit yield $(72.9 \mathrm{~kg} / \mathrm{plant})$ was observed by L-60 lowest yield of 20.52 $\mathrm{kg} /$ plant was observed in L22. Anu et al., (2015) reported similar experimental findings in mango clones of 'Langra'.

Table.1 Physical composition of different clones of mango cv. 'Langra'

\begin{tabular}{|c|c|c|c|c|c|c|c|c|}
\hline $\begin{array}{l}\text { S. } \\
\text { No }\end{array}$ & Clones & $\begin{array}{c}\text { Fruit } \\
\text { length } \\
\text { (cm) }\end{array}$ & $\begin{array}{l}\text { Fruit } \\
\text { width } \\
\text { (cm) }\end{array}$ & $\begin{array}{c}\text { Fruit } \\
\text { weight (g) }\end{array}$ & $\begin{array}{c}\text { Pulp } \\
(\%)\end{array}$ & $\begin{array}{l}\text { Peel } \\
(\%)\end{array}$ & $\begin{array}{c}\text { Stone } \\
(\%)\end{array}$ & $\begin{array}{l}\text { Fruit yield } \\
\left(\mathrm{kg} / \text { plant }^{-1}\right)\end{array}$ \\
\hline 1. & L-11 & 7.92 & 5.53 & 125.71 & 69.70 & 12.53 & 14.58 & 45.72 \\
\hline 2. & L-12 & 8.51 & 5.60 & 136.37 & 62.61 & 12.73 & 11.94 & 47.59 \\
\hline 3. & L-13 & 8.47 & 6.24 & 155.47 & 67.29 & 10.84 & 13.23 & 45.93 \\
\hline 4. & L-14 & 8.43 & 5.75 & 150.54 & 58.56 & 9.37 & 13.80 & 46.25 \\
\hline 5. & L-70 & 7.37 & 5.55 & 122.66 & 58.73 & 11.38 & 13.94 & 55.38 \\
\hline 6. & L-15 & 8.18 & 5.73 & 147.47 & 57.59 & 10.22 & 13.05 & 24.76 \\
\hline 7. & L-16 & 8.74 & 5.64 & 123.25 & 59.32 & 12.32 & 15.20 & 48.30 \\
\hline 8. & L-17 & 7.91 & 5.40 & 122.57 & 60.48 & 13.19 & 16.03 & 31.41 \\
\hline 9. & L-68 & 7.68 & 5.48 & 112.69 & 58.67 & 15.53 & 20.52 & 43.29 \\
\hline 10. & L-69 & 7.38 & 5.46 & 116.36 & 89.26 & 12.46 & 15.33 & 44.32 \\
\hline 11. & L-18 & 8.02 & 5.87 & 130.14 & 68.70 & 12.20 & 17.57 & 51.46 \\
\hline 12. & L-20 & 8.16 & 5.73 & 126.08 & 78.17 & 17.57 & 17.67 & 42.92 \\
\hline 13. & L-22 & 9.47 & 6.24 & 185.27 & 58.20 & 10.24 & 11.93 & 24.34 \\
\hline 14. & L-21 & 7.72 & 5.77 & 161.12 & 45.15 & 6.23 & 10.35 & 25.34 \\
\hline 15. & L-26 & 8.38 & 5.81 & 216.73 & 38.63 & 5.78 & 7.48 & 35.48 \\
\hline 16. & L-25 & 8.78 & 6.10 & 204.18 & 56.72 & 6.89 & 12.64 & 41.67 \\
\hline 17. & L-24 & 8.49 & 5.76 & 165.26 & 76.67 & 8.46 & 12.87 & 40.50 \\
\hline 18. & L-27 & 8.32 & 5.82 & 184.21 & 53.45 & 7.83 & 12.17 & 46.28 \\
\hline 19. & L-28 & 8.41 & 5.96 & 172.27 & 47.36 & 16.13 & 9.80 & 36.70 \\
\hline 20. & L-29 & 8.09 & 5.73 & 156.21 & 37.50 & 10.39 & 8.17 & 27.46 \\
\hline 21. & L-31 & 8.23 & 5.58 & 116.18 & 51.50 & 10.56 & 15.81 & 26.67 \\
\hline 22. & L-32 & 8.74 & 5.37 & 177.77 & 52.47 & 11.36 & 9.79 & 43.27 \\
\hline 23. & L-37 & 7.82 & 5.50 & 131.81 & 41.66 & 11.35 & 8.05 & 40.72 \\
\hline 24. & L-35 & 7.76 & 5.16 & 126.30 & 82.55 & 10.28 & 14.35 & 38.81 \\
\hline 25. & L-34 & 8.90 & 5.88 & 182.85 & 56.17 & 11.28 & 11.43 & 46.23 \\
\hline
\end{tabular}




\begin{tabular}{|l|l|l|l|c|c|c|c|c|}
\hline $\mathbf{2 6 .}$ & L-38 & 8.30 & 5.60 & 138.39 & 90.60 & 17.15 & 11.40 & 44.60 \\
\hline 27. & L-40 & 8.86 & 5.82 & 154.63 & 76.517 & 12.08 & 13.59 & 40.18 \\
\hline $\mathbf{2 8 .}$ & L-43 & 7.96 & 5.65 & 167.21 & 109.53 & 10.16 & 9.68 & 44.23 \\
\hline 29. & L-42 & 9.34 & 6.24 & 206.27 & 49.12 & 9.36 & 9.61 & 35.50 \\
\hline 30. & L-41 & 8.25 & 5.66 & 168.26 & 38.41 & 7.67 & 9.78 & 36.19 \\
\hline 31. & L-46 & 8.27 & 6.33 & 120.37 & 56.10 & 13.20 & 15.40 & 25.93 \\
\hline 32. & L-54 & 8.44 & 5.63 & 175.17 & 61.15 & 12.12 & 10.12 & 36.43 \\
\hline 33. & L-59 & 8.03 & 5.85 & 153.20 & 63.80 & 9.31 & 14.27 & 49.00 \\
\hline 34. & L-57 & 9.45 & 6.31 & 256.36 & 58.15 & 6.57 & 8.00 & 42.50 \\
\hline 35. & L-60 & 8.72 & 6.15 & 180.43 & 67.50 & 9.43 & 11.50 & 72.90 \\
\hline 36. & L-62 & 8.73 & 6.45 & 156.21 & 62.12 & 10.65 & 12.74 & 43.86 \\
\hline $\mathbf{3 7 .}$ & L-61 & 8.36 & 5.72 & 156.18 & 35.87 & 11.37 & 11.35 & 37.47 \\
\hline 38. & L-63 & 7.87 & 6.24 & 171.15 & 70.45 & 16.10 & 9.87 & 43.30 \\
\hline 39. & L-66 & 7.88 & 5.73 & 158.36 & 53.20 & 12.25 & 13.45 & 55.95 \\
\hline 40. & L-72 & 7.49 & 5.73 & 144.27 & 48.54 & 8.23 & 7.36 & 45.46 \\
\hline S Em \pm & 0.04 & 0.04 & 0.17 & 0.07 & 0.07 & 0.23 & 0.37 \\
\hline CD at 5\% & 0.13 & 0.13 & 0.58 & 0.19 & 0.22 & 0.64 & 1.04 \\
\hline
\end{tabular}

Table.2 Biochemical variation in different clones of mango cv. 'Langra'

\begin{tabular}{|c|c|c|c|c|c|c|c|}
\hline S.No & Clones & $\begin{array}{c}\text { TSS } \\
\left({ }^{0} \text { Brix }\right)\end{array}$ & $\begin{array}{c}\text { Acidity } \\
(\%)\end{array}$ & $\begin{array}{c}\text { Total sugar } \\
(\%)\end{array}$ & $\begin{array}{c}\text { Reducing sugar } \\
(\%)\end{array}$ & $\begin{array}{l}\text { Non-reducing } \\
\text { sugar }(\%)\end{array}$ & $\begin{array}{l}\text { Ascorbic acid } \\
(\mathrm{mg} / \mathbf{1 0 0 g})\end{array}$ \\
\hline 1. & L-11 & 16.00 & 0.33 & 20.10 & 5.22 & 14.88 & 80.10 \\
\hline 2. & L-12 & 21.07 & 0.33 & 19.12 & 4.73 & 14.48 & 81.23 \\
\hline 3. & L-13 & 19.97 & 0.36 & 19.67 & 5.22 & 14.38 & 81.51 \\
\hline 4. & L-14 & 17.13 & 0.26 & 20.20 & 5.12 & 15.38 & 80.47 \\
\hline 5. & $\mathrm{~L}-70$ & 23.93 & 0.34 & 19.60 & 4.70 & 14.91 & 83.20 \\
\hline 6. & L-15 & 20.00 & 0.42 & 20.13 & 5.82 & 14.24 & 80.35 \\
\hline 7. & L-16 & 19.18 & 0.43 & 19.28 & 5.53 & 13.75 & 84.37 \\
\hline 8. & L-17 & 21.17 & 0.33 & 21.09 & 6.41 & 14.70 & 81.35 \\
\hline 9. & L-68 & 20.06 & 0.32 & 18.67 & 5.34 & 13.49 & 83.74 \\
\hline 10. & L-69 & 21.53 & 0.24 & 21.59 & 5.33 & 16.26 & 80.27 \\
\hline 11. & L-18 & 17.06 & 0.36 & 20.63 & 6.56 & 14.07 & 81.32 \\
\hline 12. & $\mathrm{~L}-20$ & 23.96 & 0.30 & 20.54 & 6.13 & 14.41 & 82.50 \\
\hline 13. & $\mathrm{~L}-22$ & 21.08 & 0.50 & 21.28 & 5.44 & 15.75 & 82.47 \\
\hline 14. & $\mathrm{~L}-21$ & 20.67 & 0.52 & 21.71 & 6.83 & 14.88 & 83.77 \\
\hline 15. & L-26 & 19.16 & 0.52 & 19.17 & 4.35 & 14.97 & 85.38 \\
\hline 16. & L-25 & 19.16 & 0.50 & 20.52 & 5.40 & 15.04 & 82.77 \\
\hline 17. & L-24 & 21.97 & 0.33 & 20.63 & 5.62 & 15.02 & 82.32 \\
\hline 18. & L-27 & 22.13 & 0.23 & 21.10 & 5.98 & 15.62 & 82.51 \\
\hline 19. & L-28 & 20.29 & 0.31 & 18.21 & 5.23 & 13.07 & 85.67 \\
\hline 20. & L-29 & 21.44 & 0.23 & 15.50 & 4.32 & 11.48 & 86.64 \\
\hline 21. & L-31 & 22.46 & 0.42 & 21.14 & 5.60 & 15.54 & 81.77 \\
\hline 22. & L-32 & 21.38 & 0.22 & 20.50 & 5.87 & 14.61 & 82.74 \\
\hline 23. & L-37 & 18.21 & 0.23 & 15.28 & 3.78 & 11.62 & 86.57 \\
\hline
\end{tabular}




\begin{tabular}{|c|c|c|c|c|c|c|c|}
\hline 24. & L-35 & 17.34 & 0.32 & 20.07 & 5.18 & 14.98 & 80.25 \\
\hline 25. & L-34 & 22.07 & 0.24 & 19.56 & 4.75 & 14.81 & 82.71 \\
\hline 26. & L-38 & 18.46 & 0.23 & 22.70 & 5.34 & 17.36 & 80.33 \\
\hline 27. & L-40 & 20.18 & 0.33 & 18.40 & 4.60 & 13.88 & 84.30 \\
\hline 28. & L-43 & 21.23 & 0.44 & 19.21 & 3.46 & 15.75 & 83.32 \\
\hline 29. & L-42 & 19.07 & 0.53 & 18.41 & 4.58 & 13.83 & 82.71 \\
\hline 30. & L-41 & 19.47 & 0.35 & 19.48 & 4.53 & 14.95 & 81.55 \\
\hline 31. & L-46 & 18.10 & 0.35 & 20.72 & 5.49 & 15.33 & 83.56 \\
\hline 32. & L-54 & 17.16 & 0.37 & 18.26 & 4.49 & 13.87 & 86.20 \\
\hline 33. & L-59 & 16.62 & 0.42 & 19.36 & 3.74 & 15.62 & 82.50 \\
\hline 34. & L-57 & 21.17 & 0.43 & 18.87 & 4.23 & 14.65 & 86.82 \\
\hline 35. & L-60 & 19.63 & 0.23 & 19.78 & 5.20 & 14.62 & 80.63 \\
\hline 36. & L-62 & 19.45 & 0.43 & 20.31 & 3.77 & 16.54 & 82.45 \\
\hline 37. & L-61 & 21.00 & 0.36 & 20.63 & 5.45 & 15.28 & 84.23 \\
\hline 38. & L-63 & 22.33 & 0.22 & 20.26 & 5.53 & 14.73 & 82.22 \\
\hline 39. & L-66 & 22.47 & 0.22 & 19.50 & 4.40 & 15.11 & 83.34 \\
\hline 40. & L-72 & 18.46 & 0.25 & 19.43 & 5.51 & 14.13 & 82.69 \\
\hline \multicolumn{2}{|c|}{ S Em \pm} & 0.10 & 0.10 & 0.09 & 0.09 & 0.14 & 0.12 \\
\hline \multicolumn{2}{|c|}{ CD at $5 \%$} & 0.28 & 0.28 & 0.26 & 0.25 & 0.39 & 0.34 \\
\hline
\end{tabular}

\section{Biochemical characterization}

The highest TSS contained in the fruit of L-20 (23.96 ${ }^{0}$ Brix), L-70 (23.93 ${ }^{0}$ Brix), whereas L11 (16 ${ }^{0}$ Brix) showed the lowest (Table 2). Sixty mango cultivars were evaluated by Katrodia et al., (1989) and they noted that total soluble solids content was recorded highest in cultivar 'Alphonso' (21.5 persent), Banarasi Langra' (21.1 per cent) and 'Kesar' (21.2 per cent). The highest acidity was noted in L-21 $(0.52 \%)$ which was significantly higher compared to other clones studied. The lowest acidity was observed in L-32 $(0.2 \%)$ followed by L-63, L-66. L-38 had the highest total sugars $(22.70 \%)$, content whereas, the lowest total sugar content was observed in L$37(15.28 \%)$.

The reducing sugar content was found to be highest in L-21 (6.83\%) whereas L-43 (3.46\%) showed the lowest. L-38 had the highest non-reducing sugars (17.38\%), content whereas, the lowest non-reducing sugar content was observed in L-29 (11.48\%). Dashehari exhibited the highest amount of total sugar (21.20 percent) and non-reducing sugar (15.17 percent), while 'Langra' had the highest reducing sugar content (6.30 percent) among twenty mango cultivars studied by Kumar et al., (2001a). L-11 produced the highest ascorbic acid $(80.10 \mathrm{mg} / 100 \mathrm{~g}$ juice$)$ and proved its superiority over remaining clones analyzed. The fruit quality and nutritive value of a cultivar varies with the environmental conditions as reported by Elizabeth (1939) and Hambler et al., (1945).

In conclusion the wide variation in almost all the physico-chemical parameters was observed in forty clones of Langra $\mathrm{cv}$. Conclusively, it could be suggested that some clones L-57, L-60, L-43, L-20, L-70 etc. have great potential and can be further utilized in breeding programs.

\section{Acknowledgments}

The first author is thankful to Jawaharlal Nehru KrishiVishwaVidyalaya, Jabalpur authority for providing facilities and technical guidance during the course of investigation of $\mathrm{Ph}$. D. research work. 


\section{References}

Anonymous. 2018. Indian Horticulture Database for 2018. National Horticulture Board, DAC, Govt. of India, Gurgaon, Haryana: 9.

Anu, A., Deo, P.B., Kumar, R., Kumar P,., Patel, V.B. and Jha, R.N. 2015. Clonal variability studies in 'langra' mango (Mangifera indica L.) using morphological, biochemical and molecular markers. International Journal of Agriculture, Environment and Biotechnology 8(3): 567-581.

Badyal, J. and Bhuttani, V. P. 1989. Physicochemical characteristic of some mango cultivars under sub-mountain regions of Himachal Pradesh. Haryana J. Hort. Sci., 18: 51-55.

Elizabeth, M. 1939. Vitamin C and light. Proc. Amer. Soc. Hort. Sci. 36: 498.

Hambler, K.C. Bernstein, L. and Maynard, L.A. 1945. Effect of light intensity, day length, temperature and the other environmental factors on ascorbic acid contents of Tomatoes. J. Nutrition. 29:85.

Katrodia, J.S., Bhuva, H.P. and Patel, G.L. 1989. Yield and quality performance of mango germplasm under south Gujarat conditions. Acta Horticulture, 231:121124.

Kumar, R., Patel, M.P., Bose, U.S. and Tripathi, S.K. 2001. Physico-chemical studies of some important mango varieties of Madhya Pradesh. Crop Research Hissar, 22(1): 38-42.

Lane, J.H. and Eyon, L. 1923. Determination of reducing sugar by Fehling's solution with methylene blue as indicator. J. Soc. Chem. Ind. Trans. 42:32-36.

Ranganna, S. 2000. Handbook of Analysis of Quality control for Fruit and Vegetables Products $2^{\text {nd }}$ ed. Tata McGraw Hill Pub. Co. New Delhi.

Sharma, T.R., Nair, P.K.R. and Nema, M.K. 1990. Influence of foliar spray of Urea $\mathrm{KNO}_{3}$ and NAA on physical composition of mango cv. Langra. Punjab Hort. J., 30: 47-51.

Singh, A.R., Singh, N.D.1996. Studies on bloom biology and pollination in mango (Mangifera indica L.). Recent Horticultura., 3:4-7.

Singh, M. and Maurya, V.N. 1986. Performance of some late mango varieties in Gangetic plains of North India. Punjab J. Hort., 26:8- 12.

\section{How to cite this article:}

Megha Rajpoot, T. R. Sharma, S. K. Pandey and Mohni Parmar. 2020. Physico- chemical Variability in Clone of Mango cv Langra at Kymore Plateau and Satpura Hills of Madhya Pradesh. Int.J.Curr.Microbiol.App.Sci. 9(11): 1207-1212. doi: https://doi.org/10.20546/ijcmas.2020.911.141 